\title{
Tracking Control of a Class of Differential Inclusion Systems via Sliding Mode Technique
}

\author{
Lei-Po Liu Zhu-Mu Fu Xiao-Na Song \\ College of Electric and Information Engineering, Henan University of Science and Technology, Luoyang 471003, China
}

\begin{abstract}
The tracking problem for a class of differential inclusion systems is investigated. Using global sliding mode control approach, a tracking control is proposed such that the output of a differential inclusion system tracks the desired trajectory asymptotically. An extensive reaching law is proposed to achieve the chattering reduction. Finally, an example is given to illustrate the validity of the proposed design.
\end{abstract}

Keywords: Tracking, differential inclusion systems, global sliding mode control, chattering reduction, an extensive reaching law.

\section{Introduction}

Tracking control is always a very active research area due to its wide applications. In the past few decades, various approaches for tracking control have been presented, such as fuzzy control approach ${ }^{[1]}$, composite tracking control approach ${ }^{[2]}$, adaptive control approach ${ }^{[3]}$, sliding mode control approach ${ }^{[4]}$, etc. Because sliding mode control has attractive features such as fast response, good transient response and insensitivity to variations in system parameters and external disturbances ${ }^{[5-11]}$, it is a substantial method for the tracking control design of nonlinear systems.

Generally speaking, the differential inclusion systems are considered a generalization of differential equations, and many practical systems are described by differential inclusion systems, so the study of differential inclusion systems has been paid much attention ${ }^{[12-21]}$. In [12], a necessary and sufficient condition for the stability of polytopic linear differential inclusion systems was derived by bilinear matrix equations. In [13], the problem of tracking control of nonlinear uncertain dynamical systems described by differential inclusions was studied. In [14], a nonlinear control design method for linear differential inclusion systems was presented by using quadratic Lyapunov functions of their convex hull. Using the method in [14], Sun ${ }^{[15]}$ considered the uniformly ultimately bounded tracking control of linear differential inclusions with stochastic disturbance and Huang et al. ${ }^{[16]}$ considered the stabilization of linear differential inclusion system with time delay. In [17], a frequency-domain approach was proposed to analyze the globally asymptotic stability of differential inclusion systems with discrete and distributed time-delays. In $[18,19]$, the fundamental information about differential inclusions is introduced. But the research of tracking control of differential inclusion systems using sliding mode technique is not sufficient. In [20,21],

\footnotetext{
Regular Paper

Manuscript received December 29, 2012; revised August 2, 2013

This work was supported by National Natural Science Foundation of China (Nos. 61374077 and 61203047), fundamental research project (No. 142300410293) in the Science and Technology Department of Henan province, the science and technology research key project (No. 14A413001) in the Education Department of Henan province, innovation ability cultivation fund (No. 2014ZCX015) in Henan University of Science and Technology.
}

the authors considered the tracking problem of linear differential inclusion systems.

This paper applies global sliding mode control to study the tracking control problem for a class of differential inclusion systems. The main contributions of this paper lie in the following aspects: 1) the system model is a generalized model of those considered in $[4,8,9] ; 2)$ an extensive reaching law is proposed to design a sliding mode controller to make the error system asymptotically stable.

\section{Problem formulation}

Consider the following differential inclusion system:

$\left\{\begin{array}{l}\dot{x}_{i}(t)=x_{i+1}(t), 1 \leqslant i \leqslant n-1 \\ \dot{x}_{n}(t) \in \operatorname{co}\left\{\left[f_{j}(x)+g_{j}(x)(u(t)+w(t))\right\}, j=1,2, \cdots, N\right. \\ y(t)=x_{1}(t)\end{array}\right.$

where $x(t)=\left[x_{1}, x_{2}, \cdots, x_{n}\right]^{\mathrm{T}}$ is the system state, $c o\{\cdot\}$ denotes the convex hull of a set, $f_{j}(x)$ and $g_{j}(x)$ are smooth functions from $\mathbf{R}^{n}$ to $\mathbf{R}$ for $j=1, \cdots, N, u(t) \in \mathbf{R}$ is the control input, $w(t)$ is the bounded disturbance, i.e., $\|w(t)\| \leqslant \gamma$, with a positive constant $\gamma . y(t)$ is the output of the system. System (1) is most often applied in physical systems such as the Duffing-Holmes damped spring system, Van der Pol equation, robot systems and flexible-joint mechanisms ${ }^{[22]}$. This paper assumes that $g_{j}(x)>0$, for all $j=1,2, \cdots, N$.

The target of this paper is to design a feedback law such that the output $y(t)$ of the system (1) can track a known reference function $r(t)$.

By the conclusion established in convex analysis theory ${ }^{[21]}$, differential inclusion system (1) is equivalent to the following uncertain system:

$$
\left\{\begin{array}{l}
\dot{x}_{i}(t)=x_{i+1}(t), 1 \leqslant i \leqslant n-1 \\
\dot{x}_{n}(t)=\sum_{j=1}^{N} \alpha_{j}\left[f_{j}(x)+g_{j}(x)(u(t)+w(t))\right] \\
y(t)=x_{1}(t)
\end{array}\right.
$$

where $\alpha_{j}$ are uncertain parameters with the properties that 
$\alpha_{j} \geqslant 0$ and $\sum_{i=1}^{N} \alpha_{j}=1$

Denote $z(t)=\left[\begin{array}{c}z_{1}(t) \\ z_{2}(t) \\ \vdots \\ z_{n}(t)\end{array}\right]=\left[\begin{array}{c}y(t)-r(t) \\ \dot{y}(t)-\dot{r}(t) \\ \vdots \\ y^{(n-1)}(t)-r^{(n-1)}(t)\end{array}\right]=$

$\left[\begin{array}{c}x_{1}(t)-r(t) \\ x_{2}(t)-\dot{r}(t) \\ \vdots \\ x_{n}(t)-r^{(n-1)}(t)\end{array}\right]$,

, where $z(t)$ is called as the error of

tracking. $z(t)$ satisfies the following

$$
\left\{\begin{array}{l}
\dot{z}_{i}(t)=z_{i+1}(t), 1 \leqslant i \leqslant n-1 \\
\dot{z}_{n}(t)=\sum_{j=1}^{N} \alpha_{j}\left[f_{j}(x)+g_{j}(x)(u(t)+w(t))\right]-r^{(n)}(t) .
\end{array}\right.
$$

Then the target of the design is to find a feedback law $u(t)$ such that $z(t) \rightarrow 0$ as $t \rightarrow \infty$.

The following Lemma will be useful in this paper.

Lemma 1. Consider the following linear system

$$
\dot{x}(t)=A x(t)+B u(t)
$$

where $A$ is a Hurwitz matrix and $u(t)=\mathrm{e}^{-\lambda t} \gamma(t)$ for a positive constant $\lambda$ and a bounded function $\gamma(t)$. Then

$$
\lim _{t \rightarrow \infty} x(t)=0 \text {. }
$$

Proof. Because $A$ is a Hurwitz matrix, there exist positive constants $a$ and $b$ such that $\left\|\mathrm{e}^{A t}\right\| \leqslant a \mathrm{e}^{-b t}$. And we assume $\|\gamma(t)\| \leqslant q$, where $q \geqslant 0$ is a constant.

Solving (4), we obtain

$$
x(t)=\mathrm{e}^{A t} x(0)+\int_{0}^{t} \mathrm{e}^{A(t-\tau)} B \mathrm{e}^{-\lambda \tau} \gamma(\tau) \mathrm{d} \tau
$$

thus,

$$
\begin{aligned}
\|x(t)\| \leqslant & a \mathrm{e}^{-b t}\|x(0)\|+a q\|B\| \int_{0}^{t} \mathrm{e}^{-b(t-\tau)-\lambda \tau} \mathrm{d} \tau= \\
& \left\{\begin{array}{l}
a \mathrm{e}^{-b t}\|x(0)\|+a q\|B\| \mathrm{e}^{-b t} t, \quad \text { if } b=\lambda \\
a \mathrm{e}^{-b t}\|x(0)\|+a q\|B\| \frac{\mathrm{e}^{-\lambda t}-\mathrm{e}^{-b t}}{b-\lambda}, \quad \text { if } b \neq \lambda
\end{array}\right.
\end{aligned}
$$

where the denotation $\|\cdot\|$ may have different meanings, but we always assume that they are compatible.

From (6), obviously we get

$$
\lim _{t \rightarrow \infty} x(t)=0 \text {. }
$$

\section{Main results}

For the nonlinear system (2), the control law design consists of two phases. Firstly, an appropriate sliding surface is chosen, so that sliding mode dynamics has desired performance. Secondly, a control law is designed, which guarantees that the state of system (2) converges to the sliding surface in a finite time.

\subsection{Sliding surface design}

The sliding surface is designed to ensure the error system stable asymptotically on the sliding surface.

The sliding surface is considered as

$$
s(t)=C\left(z(t)-\mathrm{e}^{-\mu t} z(0)\right) .
$$

where $C=\left[c_{1}, c_{2}, \cdots, c_{n-1}, 1\right]$ and $c_{i}(i=1,2, \cdots, n-1)$ will be determined, $\mu>0$ is an appropriate constant, $z(0)$ is the initial state of the error.

Let $s(t)=0$. Then (8) leads to

$$
z_{n}(t)=-\sum_{i=1}^{n-1} c_{i} z_{i}+\mathrm{e}^{-\mu t} C z(0) .
$$

From (3) and (9), the sliding mode dynamics is obtained as

$$
\left\{\begin{array}{l}
\dot{z}_{i}(t)=z_{i+1}(t), \quad 1 \leqslant i \leqslant n-2 \\
\dot{z}_{n-1}(t)=-\sum_{i=1}^{n-1} c_{i} z_{i}+\mathrm{e}^{-\mu t} C z(0)
\end{array}\right.
$$

The matrix description of (10) is

$$
\dot{\bar{z}}(t)=A \bar{z}(t)+B \mathrm{e}^{-\mu t} C z(0)
$$

where $\bar{z}(t)=\left[z_{1}, z_{2}, \cdots, z_{n-1}\right]^{\mathrm{T}}, A=$

$$
\left[\begin{array}{cccccc}
0 & 1 & 0 & \cdots & \cdots & 0 \\
0 & 0 & 1 & 0 & \cdots & 0 \\
\vdots & \vdots & \vdots & \vdots & \ddots & \vdots \\
0 & 0 & 0 & 0 & \ldots & 1 \\
-c_{1} & -c_{2} & \ldots & \ldots & \ldots & -c_{n-1}
\end{array}\right], B=\left[\begin{array}{c}
0 \\
0 \\
\vdots \\
0 \\
1
\end{array}\right] .
$$

We now choose $c_{i}(i=1, \cdots, n-1)$ such that $A$ is a Hurwitz matrix, then sliding mode dynamics (10) or (11) is asymptotically stable by Lemma 1 .

\subsection{The controller design}

Before illustrating the scheme of the controller, a reaching law is introduced in this subsection.

Lemma 2. When the reaching law is adopted as

$$
\begin{aligned}
& \dot{s}(t)=-k s-\varepsilon \mathrm{e}^{-\lambda t}\|s\|^{\alpha} \operatorname{sgn}(s), \\
& k>0, \varepsilon>0, \lambda \geqslant 0,0 \leqslant \alpha<1
\end{aligned}
$$

where $\|\cdot\|$ is the Euclidean norm, the state $x(t)$ of system will reach the sliding surface $s(t)=0$ in a finite time $T$, where

$$
T=\left\{\begin{array}{l}
\frac{1}{k(1-\alpha)-\lambda} \ln \left[1+\frac{k(1-\alpha)-\lambda}{\varepsilon(1-\alpha)}\|s(0)\|^{1-\alpha}\right] \\
\text { if } k(1-\alpha) \neq \lambda \\
\frac{\|s(0)\|^{1-\alpha}}{\varepsilon(1-\alpha)}, \quad \text { if } k(1-\alpha)=\lambda .
\end{array}\right.
$$

Proof. Pre-multiplying (12) by $s^{\mathrm{T}}$, we have

$$
s^{\mathrm{T}} \dot{s}=-k\|s\|^{2}-\varepsilon \mathrm{e}^{-\lambda t}\|s\|^{\alpha+1} .
$$

We also have

$$
s^{\mathrm{T}} \dot{s}=\frac{1}{2} \frac{\mathrm{d} s^{\mathrm{T}} s}{\mathrm{~d} t}=\frac{1}{2} \frac{\mathrm{d}\|s\|^{2}}{\mathrm{~d} t}=\|s\| \frac{\mathrm{d}\|s\|}{\mathrm{d} t} .
$$


Replacing (14) by (15), we get

$$
\frac{\mathrm{d}\|s\|}{\mathrm{d} t}=-k\|s\|-\varepsilon \mathrm{e}^{-\lambda t}\|s\|^{\alpha} .
$$

Denote $z(t)=\|s\|^{1-\alpha}$, The time-derivative of $z(t)$ yields $\frac{\mathrm{d} z}{\mathrm{~d} t}=(1-\alpha)\|s\|^{-\alpha} \frac{\mathrm{d}\|s\|}{\mathrm{d} t}=-k(1-\alpha) z(t)-\varepsilon(1-\alpha) \mathrm{e}^{-\lambda t}$.

Integrating (17) from 0 to $t$, we have

$$
z(t)=\left\{\begin{array}{l}
\mathrm{e}^{-k(1-\alpha) t} z(0)-\varepsilon(1-\alpha) \mathrm{e}^{-k(1-\alpha) t} \frac{\mathrm{e}^{[k(1-\alpha)-\lambda] t}-1}{k(1-\alpha)-\lambda}, \\
\text { if } k(1-\alpha) \neq \lambda \\
\mathrm{e}^{-k(1-\alpha) t} z(0)-\varepsilon(1-\alpha) \mathrm{e}^{-k(1-\alpha) t} t, \quad \text { if } k(1-\alpha)=\lambda .
\end{array}\right.
$$

Let $s(T)=0$. Then $z(T)=0$. Through a simple calculation, we can obtain

$$
T=\left\{\begin{array}{l}
\frac{1}{k(1-\alpha)-\lambda} \ln \left[1+\frac{k(1-\alpha)-\lambda}{\varepsilon(1-\alpha)}\|s(0)\|^{1-\alpha}\right], \\
\text { if } k(1-\alpha) \neq \lambda \\
\frac{\|s(0)\|^{1-\alpha}}{\varepsilon(1-\alpha)}, \quad \text { if } k(1-\alpha)=\lambda .
\end{array}\right.
$$

Remark 1. The reaching law presented in this paper includes three cases:

1) if $k=0, \lambda=0,0<\alpha<1$, then $\dot{s}(t)=-\varepsilon\|s\|^{\alpha} \operatorname{sgn}(s)$;

2) if $\lambda=0, \alpha=0$, then $\dot{s}(t)=-k s-\varepsilon \operatorname{sgn}(s)$;

3) if $\alpha=0$, then $\dot{s}(t)=-k s-\varepsilon \mathrm{e}^{-\lambda t} \operatorname{sgn}(s)$.

The reaching law 1) is known as power rate reaching law $\left.{ }^{[5]}, 2\right)$ is widely used in many papers, for examples in $[7-10], 3)$ is presented in [11]. The term $\mathrm{e}^{-\lambda t}$ is added to reduce the chattering phenomenon in this paper.

Remark 2. The right term of the reaching law presented in this paper is continuous in $s=0$, but in [6], the reaching law $\dot{s}(t)=-\left(\mu+\eta \mathrm{e}^{-\lambda t}\|s\|^{-\alpha}\right) s$ is not continuous in $s=0$ when $0<\alpha<1$.

From the discussion above, the sliding mode controller design is given in the following theorem.

Theorem 1. For system (1), if $u(t)$ is designed as

$$
\begin{aligned}
u(t)= & -\frac{1}{\underline{g}(x)}\left[\sum_{i=1}^{n-1} c_{i} z_{i+1}+\mu \mathrm{e}^{-\mu t} C z(0)-r^{(n)}(t)+\right. \\
& \left.k s+\left(\eta(x)+\varepsilon \mathrm{e}^{-\lambda t}|s|^{\alpha}\right) \operatorname{sgn}(s)\right]
\end{aligned}
$$

where

$$
\begin{aligned}
& \eta(x)=\left(\frac{\bar{g}(x)}{\underline{g}(x)}-1\right)\left|\sum_{i=1}^{n-1} c_{i} z_{i+1}+\mu \mathrm{e}^{-\mu t} C z(0)-r^{(n)}(t)\right|+ \\
& \bar{f}(x)+\bar{g}(x) \gamma \\
& \underline{g}(x)= \min \left\{g_{1}(x), \cdots, g_{N}(x)\right\}>0 \\
& \bar{g}(x)= \max \left\{g_{1}(x), \cdots, g_{N}(x)\right\} \\
& \bar{f}(x)= \max \left\{\left|f_{1}(x)\right|, \cdots,\left|f_{N}(x)\right|\right\} \\
& k>0, \quad \varepsilon>0, \quad \lambda \geqslant 0, \quad 1>\alpha \geqslant 0
\end{aligned}
$$

then the trajectory of the error system (3) converges to the sliding surface $s(t)=0$ in a finite time $T$ and remains on it thereafter.

Proof. Consider a Lyapunov function candidate as follows:

$$
V(t)=\frac{1}{2} s^{2}
$$

Calculating the time derivative of $V(t)$ with the trajectory of (3), we have

$$
\begin{aligned}
& \dot{V}(t)= s\left\{\sum_{i=1}^{n-1} c_{i} z_{i+1}+\mu \mathrm{e}^{-\mu t} C z(0)-r^{(n)}(t)+\right. \\
&\left.\sum_{j=1}^{N} \alpha_{j}\left[f_{j}(x)+g_{j}(x)(u(t)+w(t))\right]\right\}= \\
&\left(\sum_{j=1}^{N} \alpha_{j} g_{j}(x)\right)\left(\sum_{i=1}^{n-1} c_{i} z_{i+1}+\mu \mathrm{e}^{-\mu t} C z(0)-\right. \\
&\left.\sum^{(n)}(t)\right)-\frac{\sum_{j=1}^{N} \alpha_{j} g_{j}(x)}{\underline{g}(x)} k s^{2}+s \sum_{j=1}^{N} \alpha_{j}\left[f_{j}(x)+\right. \\
&\left.g_{j}(x) w(t)\right]-\frac{\sum_{j=1}^{N} \alpha_{j} g_{j}(x)}{g(x)}\left(\eta(x)+\varepsilon \mathrm{e}^{-\lambda t}|s|^{\alpha}\right)|s| \leqslant \\
&|s| \cdot\left[\left(\frac{\bar{g}(x)}{g(x)}-1\right) \mid \sum_{i=1}^{n-1} c_{i} z_{i+1}+\mu \mathrm{e}^{-\mu t} C z(0)-\right. \\
&\left.r^{(n)}(t) \mid+\bar{f}(x)+\bar{g}(x) \gamma\right]-k s^{2}-\left(\eta(x)+\varepsilon \mathrm{e}^{-\lambda t}|s|^{\alpha}\right)|s| .
\end{aligned}
$$

Define

$$
\begin{aligned}
\eta(x)= & \left(\frac{\bar{g}(x)}{\underline{g}(x)}-1\right)\left|\sum_{i=1}^{n-1} c_{i} z_{i+1}+\mu \mathrm{e}^{-\mu t} C z(0)-r^{(n)}(t)\right|+ \\
& \bar{f}(x)+\bar{g}(x) \gamma .
\end{aligned}
$$

Substituting (23) into (22) yields to

$$
\dot{V}(t) \leqslant-k s^{2}-\varepsilon \mathrm{e}^{-\lambda t}|s|^{\alpha+1} .
$$

By Lemma 2 and the sliding mode theory, we conclude that the trajectory of the error system (3) converges to the sliding surface $s(t)=0$ in a finite time $T$ and remains in it thereafter.

Remark 3. In Theorem 1, we apply the initial condition $z(0)$ to design the controller $(20)$, the technique is adopted by $[6]$.

\section{Numerical example}

In this section, a simulation of a nonlinear system is provided to verify the effectiveness of the method proposed in 
this paper. System model is described by

$$
\left\{\begin{array}{l}
\dot{x_{1}}=x_{2} \\
\dot{x_{2}}=\alpha_{1} x_{1}+\alpha_{2} x_{2}^{2}+\left[\alpha_{1}+\alpha_{2}\left(1+x_{2}^{2}\right)\right](u+w(t)) \\
y(t)=x_{1}(t)
\end{array}\right.
$$

where $f_{1}(x)=x_{1}, f_{2}(x)=x_{2}^{2}, g_{1}(x)=1, g_{2}(x)=1+$ $x_{2}^{2}, w(t)=0.2 \sin t$ and $\alpha_{1}+\alpha_{2}=1, \alpha_{1}, \alpha_{2} \geqslant 0$.

We choose $c_{1}=10$ such that the eigenvalue of the matrix $A$ is $\xi=-10$. The initial value $x(0)=[1-1]^{\mathrm{T}}$ and the reference function is $r(t)=\sin (t)$. The sliding surface is designed as $s(t)=10 x_{1}(t)+x_{2}(t)-10 \sin (t)-\cos (t)-8 \mathrm{e}^{-t}$. The parameters in the controller (20) are chosen as $\mu=\varepsilon=$ $1, \lambda=2, k=8, \alpha=0.5$.

Figs. 1 and 2 show the trajectories of the output state $y(t)$ and the reference function $\sin (t)$ with the different parameters $\alpha_{1}, \alpha_{2}$. From Figs. 3 and 4 , we can conclude that the method proposed in this paper reduces the chattering phenomenon. So the robustness and stability of system are improved.

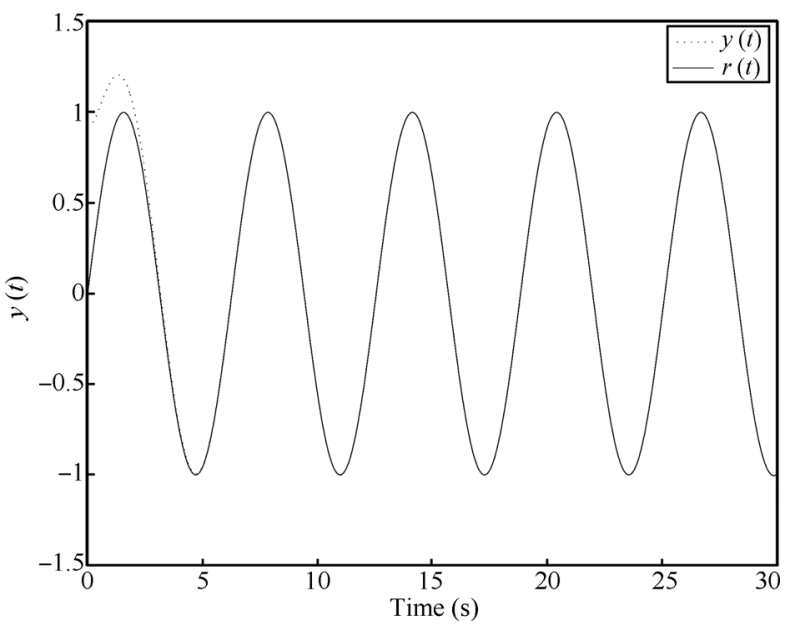

Fig. 1 Output $y(t)$ and the reference function $r(t)\left(\alpha_{1}=\alpha_{2}=\right.$ $0.5)$

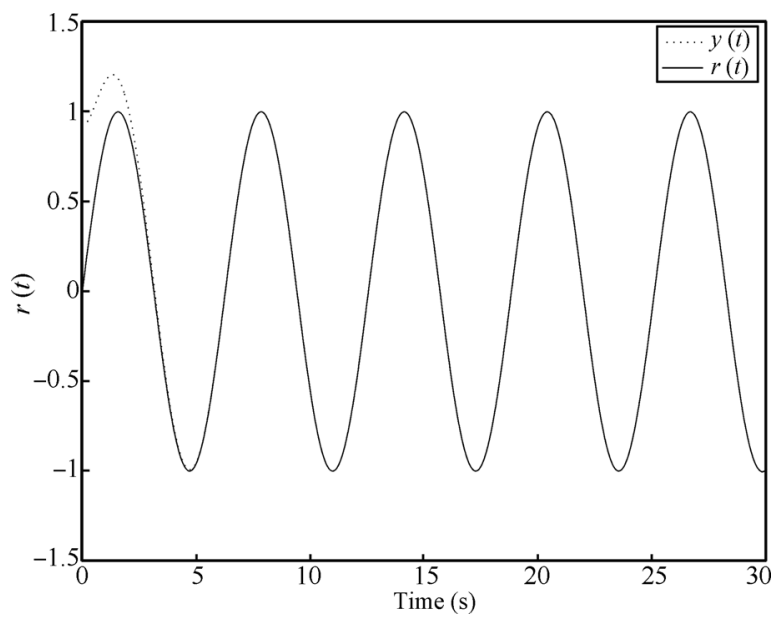

Fig. 2 Output $y(t)$ and the reference function $r(t)\left(\alpha_{1}=\right.$ $\left.0.9, \alpha_{2}=0.1\right)$

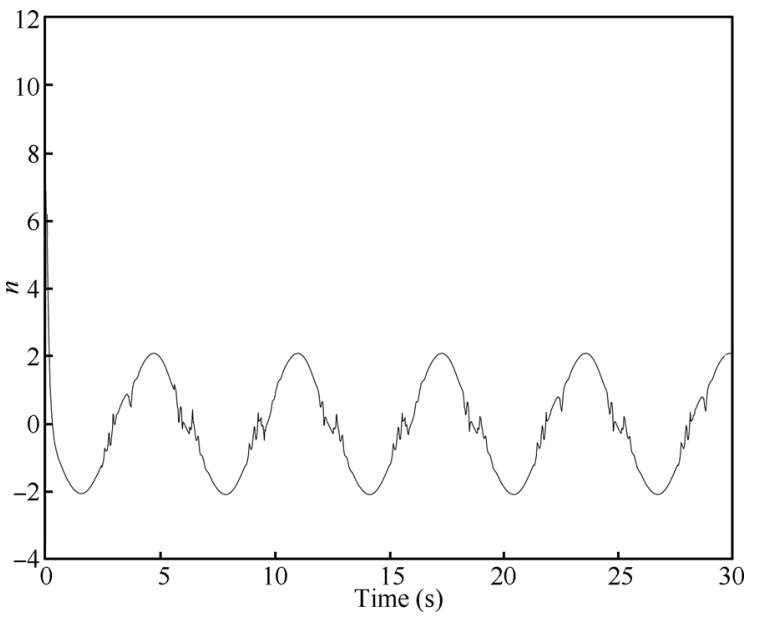

Fig. 3 Control input $u(t)\left(\alpha_{1}=0.9, \alpha_{2}=0.1\right)$

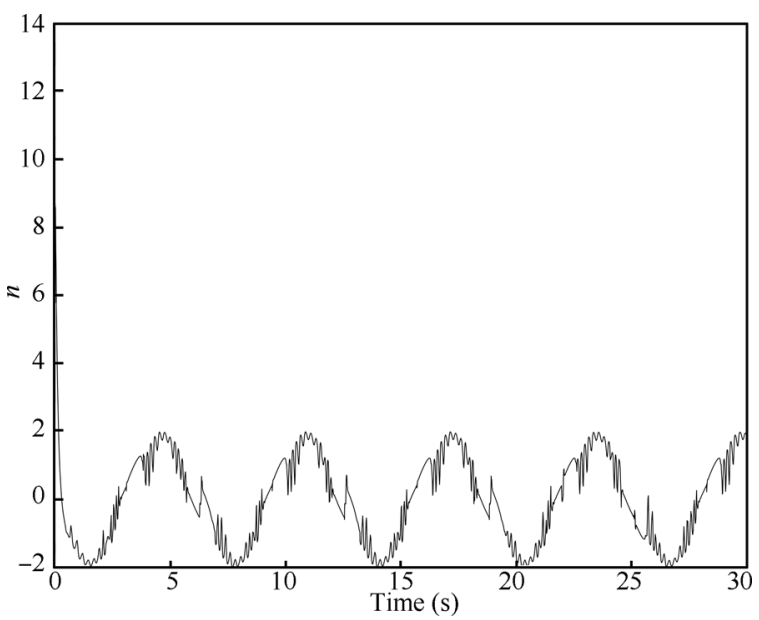

Fig. 4 Traditional sliding mode control input $u(t)$ adopted by $[7-10]\left(\alpha_{1}=0.9, \alpha_{2}=0.1\right)$

\section{Conclusion}

The tracking control for a class of differential inclusion systems has been investigated. Using global sliding mode control, a novel controller is designed to make the output of differential inclusion systems track the desired trajectory asymptotically. Finally, an example illustrates the validity of the proposed method.

\section{References}

[1] H. F. Ho, Y. K. Wong, A. B. Rad. Robust fuzzy tracking control for robotic manipulators. Simulation Modelling Practice and Theory, vol. 15, no. 7, pp. 801-816, 2007.

[2] Y. J. Sun. Composite tracking control for a class of uncertain nonlinear control systems with uncertain deadzone nonlinearities. Chaos, Solitons \& Fractals, vol. 35, no. 2 , pp. 383-389, 2008.

[3] X. Gong, Z. C. Hou, C. J. Zhao, Y. Bai, Y. T. Tian. Adaptive backstepping sliding mode trajectory tracking control for a quad-rotor. International Journal of Automation and Computing, vol. 9, no. 5, pp. 555-560, 2012.

[4] H. H. Tsai, C. C. Fuh, C. N. Chang. A robust controller for chaotic systems under external excitation. Chaos, Solitons \& Fractals, vol. 14, no. 4, pp. 627-632, 2002. 
[5] L. G. Wu, W. C. H. Daniel, C. W. Li. Sliding mode control of switched hybrid systems with stochastic perturbation. Systems \& Control Letters, vol. 60, no. 8, pp. 531-539, 2011.

[6] L. P. Liu, Z. Z. Han, W. L. Li. Global sliding mode control and application in chaotic systems. Nonlinear Dynamics, vol. 56, no. 1-2, pp. 193-198, 2009.

[7] J. M. Nazzal, A. N. Natsheh. Chaos control using sliding mode theory. Chaos, Solitons \& Fractals, vol. 33, no. 2, pp. 695-702, 2007.

[8] H. Salarieh, A. Alasty. Chaos control in uncertain dynamical systems using nonlinear delayed feedback. Chaos, Solitons \& Fractals, vol. 41, no. 1, pp. 67-71, 2009.

[9] H. T. Yau. Design of adaptive sliding mode controller for chaos synchronization with uncertainties. Chaos, Solitons \& Fractals, vol. 22, no. 2, pp. 341-347, 2004.

[10] L. P. Liu, Z. M. Fu, X. N. Song. Sliding mode control with disturbance observer for a class of nonlinear systems. International Journal of Automation and Computing, vol.9, no. 5, pp. 487-491, 2012.

[11] W. L. Li, Y. Z. Song. Chaos anti-control of nonlinear system with uncertainties. Acta Physica Sinica, vol. 57, no. 1, pp. 51-55, 2008. (in Chinese)

[12] A. P. Molchanov, Y. S. Pyatnitskiy. Criteria of asymptotic stability of differential and difference inclusions encountered in control theory. Systems \& Control Letters, vol. 13, no. 1, pp. 59-64, 1989.

[13] J. W. Chen. Asymptotic stability for tracking control of nonlinear uncertain dynamical systems described by differential inclusions. Journal of Mathematical Analysis and Applications, vol. 261, no. 1, pp. 369-389, 2001.

[14] T. S. Hu. Nonlinear control design for linear differential inclusions via convex hull of quadratics. Automatica, vol. 43, no. 4, pp. 685-692, 2007.

[15] Y. J. Sun. Stability criteria for a class of differential inclusion systems with discrete and distributed time delays. Chaos, Solitons \& Fractals, vol. 39, no. 5, pp. 2386-2391, 2007.

[16] J. Huang, Z. Z. Han, X. S. Cai, L. P. Liu. Uniformly ultimately bounded tracking control of linear differential inclusions with stochastic disturbance. Mathematics and Computers in Simulation, vol. 81, no. 12, pp. 2662-2672, 2011.

[17] L. P. Liu, Z. Z. Han, X. S. Cai, J. Huang. Robust stabilization of linear differential inclusion system with time delay. Mathematics and Computers in Simulation, vol.80, no. 5, pp. 951-958, 2010.

[18] X. S. Cai, Y. Liu, W. Zhang. Asymptotic tracking control for a class of reference signals for linear differential inclusions. International Journal of Systems Science, 2012. (Online first).
[19] X. S. Cai, J. Huang, Q. Y. Xie. Output tracking and disturbance rejection of linear differential inclusion systems. International Journal of Systems Science, vol.43, no. 11, pp. 2072-2078, 2012.

[20] S. Boyd, L. El Ghaoui, E. Feron, V. Balakrishnan. Linear Matrix Inequalities in System and Control Theory, Philadelphia: Society for Industrial and Applied Mathematics, 1994.

[21] G. V. Smirnov. Introduction to the Theory of Differential Inclusions, Providence, Rhode Island: American Mathematical Society, 2002.

[22] J. E. Slotine, W. P. Li. Applied Nonlinear Control, New Jersey: Prentice-Hall, 1991.

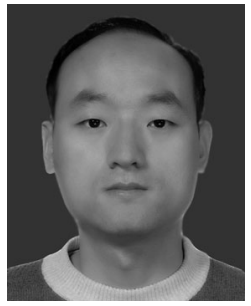

Lei-Po Liu graduated from Henan Normal University, China in 2004. He received the M. Sc. degree from Henan Normal University in 2007 and the Ph. D. degree in control theory and control engineering from Shanghai Jiao Tong University, China in 2011. He is currently an associate professor in Henan University of Science and Technology, China.

His research interests include sliding mode control, robust control and differential inclusion systems.

E-mail: liuleipo123@163.com (Corresponding author)

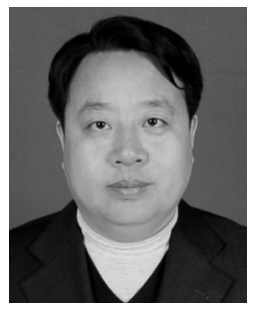

Zhu-Mu Fu received his $\mathrm{Ph}$. D. degree in control theory and control engineering from Southeast University, China in 2007. Now he is an associate professor in Henan University of Science and Technology, China.

His interest includes switch system and nonlinear control.

E-mail: fzm1974@163.com

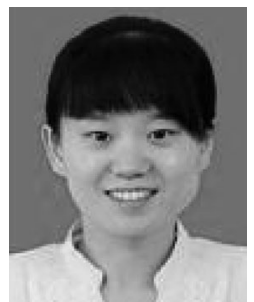

Xiao-Na Song received her Ph. D. degree in control theory and control engineering from Nanjing University of Science and Technology, China in 2011. Now she is an associate professor in Henan University of Science and Technology, China.

Her interest includes fuzzy system and robust control.

E-mail: xiaona_97@163.com 